\title{
A Decade of MWC 758 Disk Images: Where Are the Spiral-arm-driving Planets?
}

\author{
Bin Ren (任彬) ${ }^{1}$ (1), Ruobing Dong (董若冰 $)^{2,13}$ (10), Thomas M. Esposito ${ }^{3}$ (iD), Laurent Pueyo ${ }^{4}$,

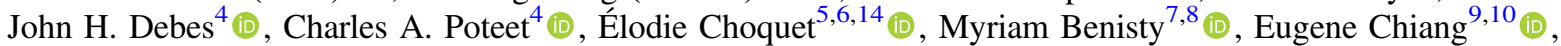 \\ Carol A. Grady ${ }^{11}$, Dean C. Hines ${ }^{4}$ (D), Glenn Schneider ${ }^{12}$ (D), and Rémi Soummer ${ }^{4}$ (D) \\ ${ }^{1}$ Department of Physics and Astronomy, The Johns Hopkins University, Baltimore, MD 21218, USA; ren@jhu.edu \\ ${ }^{2}$ Steward Observatory, University of Arizona, Tucson, AZ, 85719, USA; rdong@email.arizona.edu \\ ${ }^{3}$ Department of Astronomy, University of California, Berkeley, CA, 94720, USA; tesposito@ berkeley.edu \\ ${ }^{4}$ Space Telescope Science Institute (STScI), Baltimore, MD 21218, USA; pueyo@stsci.edu \\ ${ }^{5}$ Department of Astronomy, California Institute of Technology, 1200 E. California Boulevard, Pasadena, CA 91125, USA \\ ${ }^{6}$ Jet Propulsion Laboratory, California Institute of Technology, 4800 Oak Grove Drive, Pasadena, CA 91109, USA \\ ${ }^{7}$ Unidad Mixta Internacional Franco-Chilena de Astronomía, CNRS/INSU UMI 3386 \\ and Departamento de Astronomía, Universidad de Chile, Casilla 36-D, Santiago, Chile \\ ${ }^{8}$ Université Grenoble Alpes, CNRS, IPAG, F-38000 Grenoble, France \\ ${ }^{9}$ Department of Astronomy, University of California at Berkeley, Campbell Hall, Berkeley, CA 94720-3411, USA \\ ${ }^{10}$ Department of Earth and Planetary Science, University of California at Berkeley, McCone Hall, Berkeley, CA 94720-3411, USA \\ ${ }^{11}$ Exoplanets and Stellar Astrophysics Laboratory, Code 667, Goddard Space Flight Center Greenbelt, MD 20771, USA \\ ${ }^{12}$ Steward Observatory, The University of Arizona, Tucson, AZ 85721, USA \\ Received 2017 December 22; revised 2018 March 16; accepted 2018 March 18; published 2018 April 12
}

\begin{abstract}
Large-scale spiral arms have been revealed in scattered light images of a few protoplanetary disks. Theoretical models suggest that such arms may be driven by and corotate with giant planets, which has called for remarkable observational efforts to look for them. By examining the rotation of the spiral arms for the MWC 758 system over a 10 year timescale, we are able to provide dynamical constraints on the locations of their perturbers. We present reprocessed Hubble Space Telescope (HST)/NICMOS F110W observations of the target in 2005, and the new Keck/NIRC2 L'-band observations in 2017. MWC 758's two well-known spiral arms are revealed in the NICMOS archive at the earliest observational epoch. With additional Very Large Telescope (VLT)/SPHERE data, our joint analysis leads to a pattern speed of $0.6_{-0.6}^{+33} \mathrm{yr}^{-1}$ at $3 \sigma$ for the two major spiral arms. If the two arms are induced by a perturber on a near-circular orbit, its best-fit orbit is at 89 au $(0 . \prime 59)$, with a $3 \sigma$ lower limit of 30 au (0!'20). This finding is consistent with the simulation prediction of the location of an arm-driving planet for the two major arms in the system.
\end{abstract}

Key words: protoplanetary disks - stars: imaging - stars: individual (MWC 758)

\section{Introduction}

Planets form in gaseous and dusty protoplanetary disks around young stars that are a few million years old. Forming planets gravitationally interact with the host disk, producing structures such as gaps, spiral arms, and vortices (Kley \& Nelson 2012). By comparing observations with theoretical models, spatially resolved disk structures may yield rich information about the properties of embedded planets, such as their orbits, and dynamical constraints on their masses.

In the past decade, near-infrared imaging of disks with high spatial resolution has discovered spiral arms at tens of astronomical units in a few systems (e.g., SAO 206462: Muto et al. 2012; Garufi et al. 2013; Stolker et al. 2016; LkH $\alpha 330$ : Akiyama et al. 2016; MWC 758: Grady et al. 2013; Benisty et al. 2015; HD 100453: Wagner et al. 2015; Benisty et al. 2017; and HD 141569 A: Mouillet et al. 2001; Clampin et al. 2003; Konishi et al. 2016). Hydrodynamical and radiative transfer simulations have suggested two mechanisms for reproducing such structure: gravitational instability (Lodato \& Rice 2005; Dong et al. 2015a), which occurs in disks with sufficient mass (Kratter \& Lodato 2016), and companion-disk

\footnotetext{
${ }^{13}$ Bok Fellow.

${ }^{14}$ Hubble Fellow.
}

interaction (Dong et al. 2015b; Zhu et al. 2015; Bae et al. 2016). Because the host disks in these few systems are probably not massive enough to trigger the gravitational instability (e.g., Andrews et al. 2011), the latter scenario is more likely.

Detailed numerical simulations have quantified the dependencies of arm separation and contrast on the companion mass and disk properties (Fung \& Dong 2015; Dong \& Fung 2017). These relations have been used to infer the parameters of hypothesized arm-driving companions. A proof of concept of this mechanism has recently been provided by the HD 100453 system, where both the arms and the companion have been found, with their physical connections numerically supported (Dong et al. 2016b; Wagner et al. 2018). Extensive direct imaging observations have been carried out to look for the predicted arm-driving companions in a few other systems. Assuming hot-start planet formation models (e.g., Baraffe et al. 2015), they have generally ruled out all but planetary mass objects of a few Jupiter masses or less (e.g., Maire et al. 2017).

Companion-driven arms corotate with their driver. Therefore, by measuring their pattern speed, the orbital period, and thus semimajor axis, of their companion can be constrained (e.g., Lomax et al. 2016). We perform such an exercise for the spiral-arm system MWC 758, taking advantage of observations of the arms over a decade-long baseline established by a 2005 
HST/NICMOS observation and 2015/2017 VLT/SPHERE and Keck/NIRC2 observations.

MWC 758 is a Herbig Ae star located at $151_{-9}^{+8}$ pc (Gaia Collaboration et al. 2016) with an age of $3.5 \pm 2.0 \mathrm{Myr}$ (Meeus et al. 2012), and mass of $\sim 2.0 M_{\odot} .^{15}$ The disk has a low inclination of $\sim 20^{\circ}$ (Isella et al. 2010). Its two prominent, roughly $180^{\circ}$ rotationally symmetric arms were first discovered with Subaru/HiCIAO (Grady et al. 2013), and subsequently characterized in detail with $V L T /$ SPHERE (Benisty et al. 2015 ), with a third arm and point-source candidate at $\sim 0$ !" 11 (17 au) recently reported in Reggiani et al. (2017) using Keck/ NIRC2. Numerical simulations by Dong et al. (2015a) suggested that both arms can be produced by a multi-Jupitermass planet at $\sim 0$ " 6 from the star.

\section{Data Acquisition and Reduction}

In this section, we describe the observations and data reduction for our 2005 HST and 2017 Keck programs.

\subsection{NICMOS}

The HST/NICMOS coronagraphic instrument observed the MWC 758 system in total intensity with the F110W filter $\left(\lambda_{\text {cen }}=1.1 \mu \mathrm{m}\right.$ ) on 2005 January 7 (Proposal ID: 10177, PI: G. Schneider), and the unresolved disk morphology was presented in Grady et al. (2013). To retrieve the morphology of the spiral arms, we obtain calibrated NICMOS images of MWC 758 and another 814 reference star exposures, i.e., point-spread functions (PSFs), from the Archival Legacy Investigations for Circumstellar Environments (ALICE) project (PI: R. Soummer; Choquet et al. 2014; Hagan et al. 2018). We align the observations for better astrometry by employing a Radon-transform-based technique (Pueyo et al. 2015; Poteet et al. 2018), which focuses on the diffraction spikes in each exposure. To minimize color mismatch, telescope breathing, and cold mask alignment, we select the 81 closest PSFs in the $L^{2}$-norm sense, and perform PSF subtraction with the Non-negative Matrix Factorization (NMF) method (Ren et al. 2018), which is shown to preserve the morphology of circumstellar disks better, especially in reference differential imaging scenarios.

In Figure 1, we present the reduction results of the NICMOS observations at two telescope orientations (three exposures each) and their signal-to-noise $(\mathrm{S} / \mathrm{N})$ maps. We argue the physical existence of the detection since the spiral pattern is (1) consistent within the same telescope orientation, as shown from the $\mathrm{S} / \mathrm{N}$ maps, which are calculated from dividing the combined result by the pixel-wise standard deviation of the ones constituing them; (2) independent of telescope orientation $\left(30^{\circ}\right.$ separation), which excludes the scenario of unsuccessful noise removal; (3) not depending on the number of NMF components, reducing the possibility of underfitting and overfitting; (4) not resembling the null detections in the ALICE archive, as well as a reduction consistency using a principalcomponent-analysis-based reduction method (Soummer et al. 2012).

The integrated flux for $0 . ! 3<r<0$ ". 5 is $2.0 \pm 0.8 \mathrm{mJy}$ at $1 \sigma$ level, consistent with the upper limit reported in Grady et al. (2013). We notice flux variations between the two telescope

\footnotetext{
${ }^{15}$ We derive the stellar mass from the Siess et al. (2000) pre-main-sequence evolutionary tracks, assuming stellar effective temperature and luminosity of $7580 \mathrm{~K}$ and $19.6 L_{\odot}$ (van der Marel et al. 2016; after scaling the stellar luminosity using the new Gaia distance).
}

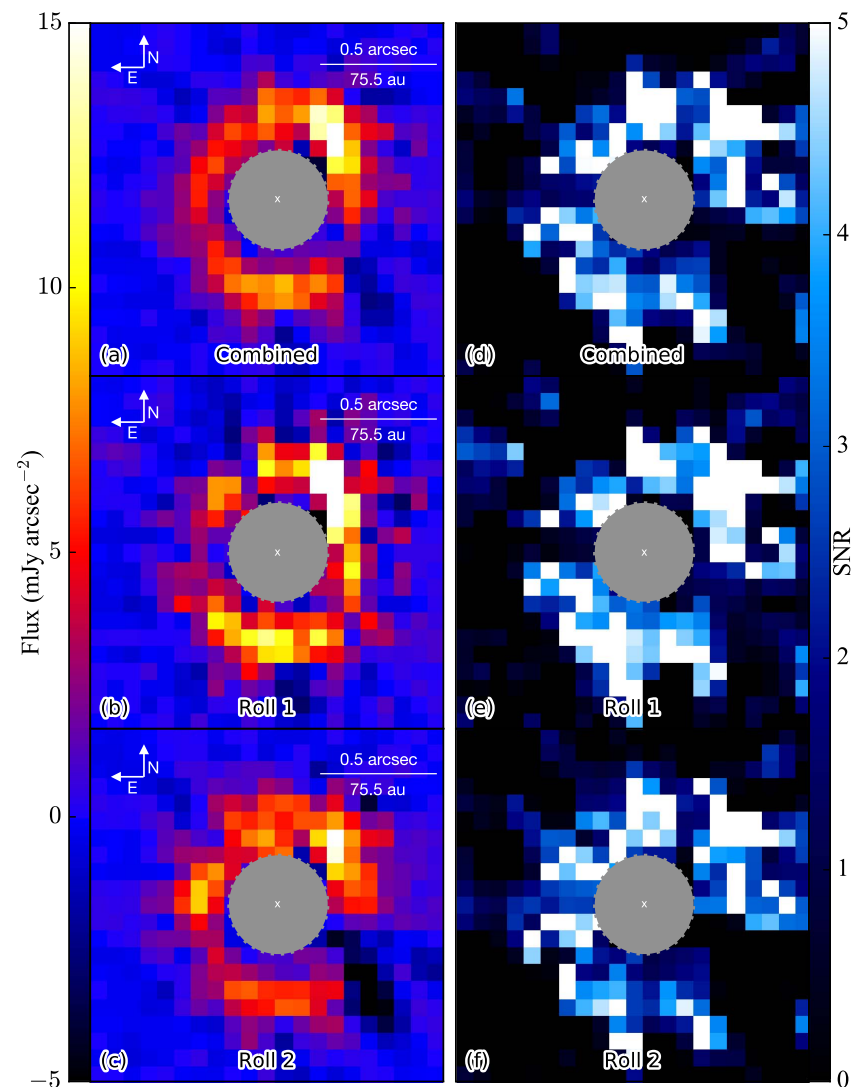

Figure 1. Left: the NICMOS images of MWC 758, including the combined (a) and two different rolls in (b, c). Right: the $\mathrm{S} / \mathrm{N}$ maps, calculated from dividing the final images by the pixel-wise standard deviation of their constituting ones. The inner working angles are marked with gray circles, and stellar locations with white crosses.

orientations; however, we do not address the origin of this difference in this letter, but focus on the morphology only.

\section{2. $N I R C 2$}

We observed MWC 758 with Keck/NIRC2 in $L^{\prime}$-band total intensity $\left(\lambda_{\text {cen }}=3.8 \mu \mathrm{m}\right)$ on 2017 February 2 (PI: E. Chiang). The data were obtained with the narrow camera $\left(9.971\right.$ mas pixel ${ }^{-1}$; Service et al. 2016) in "vertical angle mode" to allow for angular differential imaging (ADI; Marois et al. 2006). Our observations totaled 262 images, each consisting of 30 coadds of $1.0 \mathrm{~s}$ exposures, covering $161^{\circ}$ of field rotation. Airmass varied from 1.01 to 1.39 and precipitable water vapor was approximately $2.5 \mathrm{~mm}$.

The vector vortex coronagraph (Serabyn et al. 2017) was used in combination with the existing Keck II adaptive optics system to suppress host star light. The QACITS control system (Huby et al. 2017) maintained alignment of the vortex mask with the star during observations, and images are aligned with each other to sub-pixel precision using a downhill simplex algorithm to minimize residuals of the stellar PSF in frames differenced with a selected reference frame. Calibrated images are produced from raw images by performing dark subtraction, flat-fielding, thermal background subtraction with dedicated sky frames, and distortion correction (Service et al. 2016). The absolute star positions are then determined to 0.5 pixel precision in both spatial dimensions by a Radon transform of the averaged frames (Pueyo et al. 2015). 


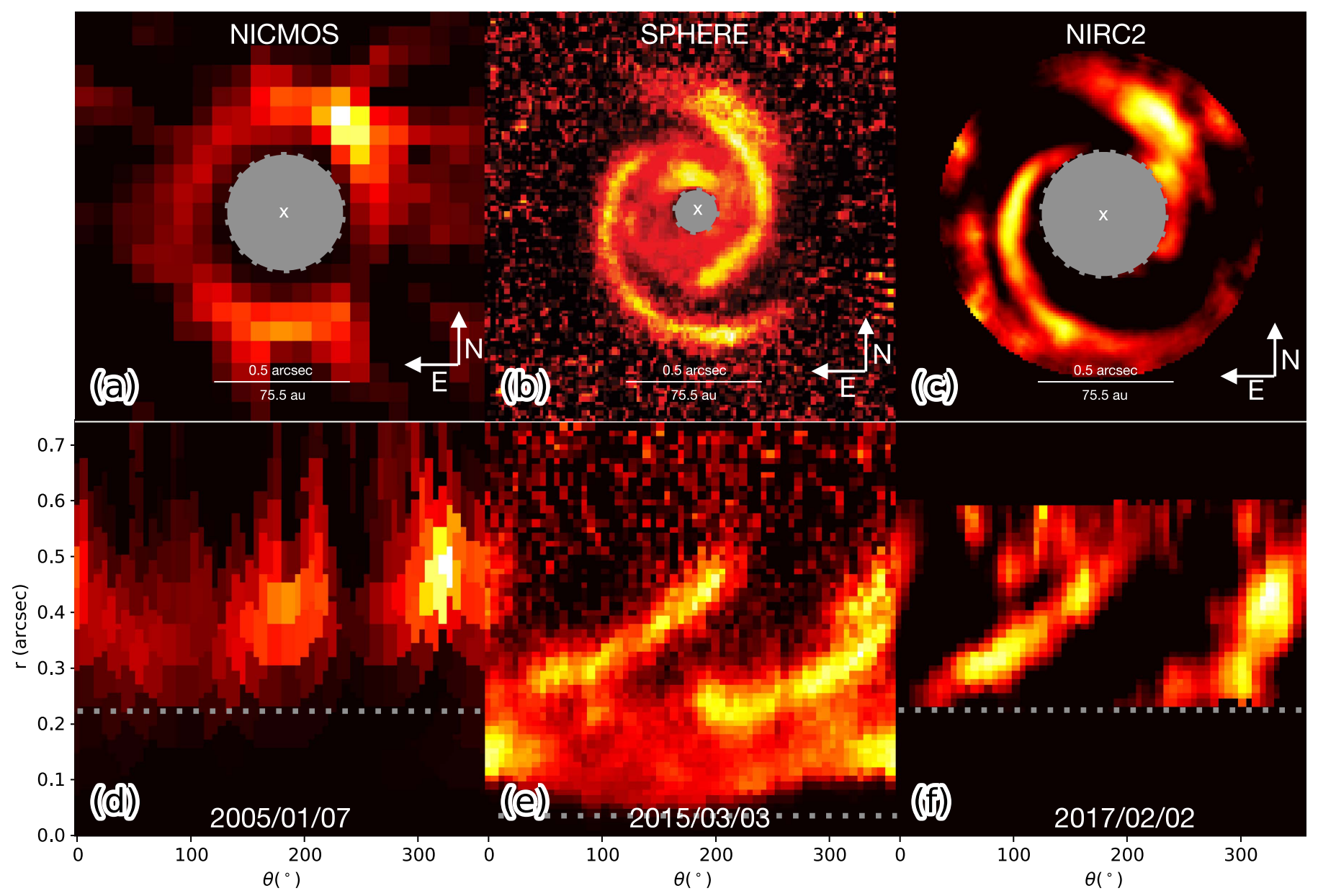

Figure 2. The $r^{2}$-scaled NICMOS, SPHERE, and NIRC2 (from left to right) observations of MWC 758 in Cartesian (top) and polar (bottom) coordinates with total flux normalized to unity. The gray dotted circles and lines mark the inner working angles.

We subtract the stellar PSF from the calibrated images using the NMF method. PSF-subtraction algorithms with ADI are known to distort the morphology of extended objects from selfsubtraction (e.g., Follette et al. 2017); therefore, for each image, this bias is avoided by a minimum rotation threshold of $45^{\circ}$ for the selection of its reference images; the final result is then the median of the PSF-subtracted images.

\subsection{SPHERE}

We obtain the $V L T$ /SPHERE polarized intensity result in $Y$-band $\left(\lambda_{\text {cen }}=1.04 \mu \mathrm{m}\right)$ on 2015 March 3 from Benisty et al. (2015).

\section{Data Analysis}

To measure the pattern speed of the arms, we first scale the surface brightnesses of the reduced NICMOS, NIRC2, and SPHERE images by the distance-dependent factor $r^{2}$. The results in Cartesian and polar coordinates are presented in Figure 2.

There are three main differences among our observational data sets: (1) the pixel size of the NICMOS instrument is $\sim 8$ times larger than the other two; (2) the NICMOS and NIRC2 observations measure the total intensity, while SPHERE traces the polarized light, and (3) the NICMOS and SPHERE observations are at $\sim 1 \mu \mathrm{m}$, while the NIRC2 observation is at $\sim 3.8 \mu \mathrm{m}$. For (1), we interpolate the NICMOS image to match the pixel scales of the others. For (2), we reduced the SPHERE ADI total intensity observation, compared it with the polarized map, and found no discernible discrepancy; this agreement is also endorsed by the simulation in Dong et al. (2016a). For (3), we compare observations at roughly the same central wavelength $\left(\lambda_{\text {cen }}\right)$.

\subsection{Measurement of Rotation of Spirals}

\subsubsection{NIRC2: 2015 versus 2017 (1.28 years apart)}

To mitigate any systematic offset between instruments, and provide an initial constraint on the rotation, we compare two observations from the same Keck/NIRC2 instrument: our 2017 February 02 observation, and the 2015 October 24 observation (PI: E. Serabyn, Reggiani et al. 2017), which is aligned and reduced with an identical procedure.

We quantify the rotation of the spiral arms as follows: in polar coordinates, we first fit Gaussian profiles to the brightness of the spiral arms at a fixed radial separation; then for each arm, we perform the weighted Least Square Dummy Variable (LSDV, Appendix) analysis to fit the same morphological profile in both epochs to obtain their relative rotation. For the southern and northern primary arms (marked with "1" and "3" in Figure 3), we obtain a rotation of $\Delta \theta_{S}^{(1.28 \text { year })}=0^{\circ} .77 \pm 10^{\circ} .65,{ }^{16}$ and

\footnotetext{
${ }^{16}$ The calculated uncertainty in this Letter is $3 \sigma$ unless otherwise specified.
} 


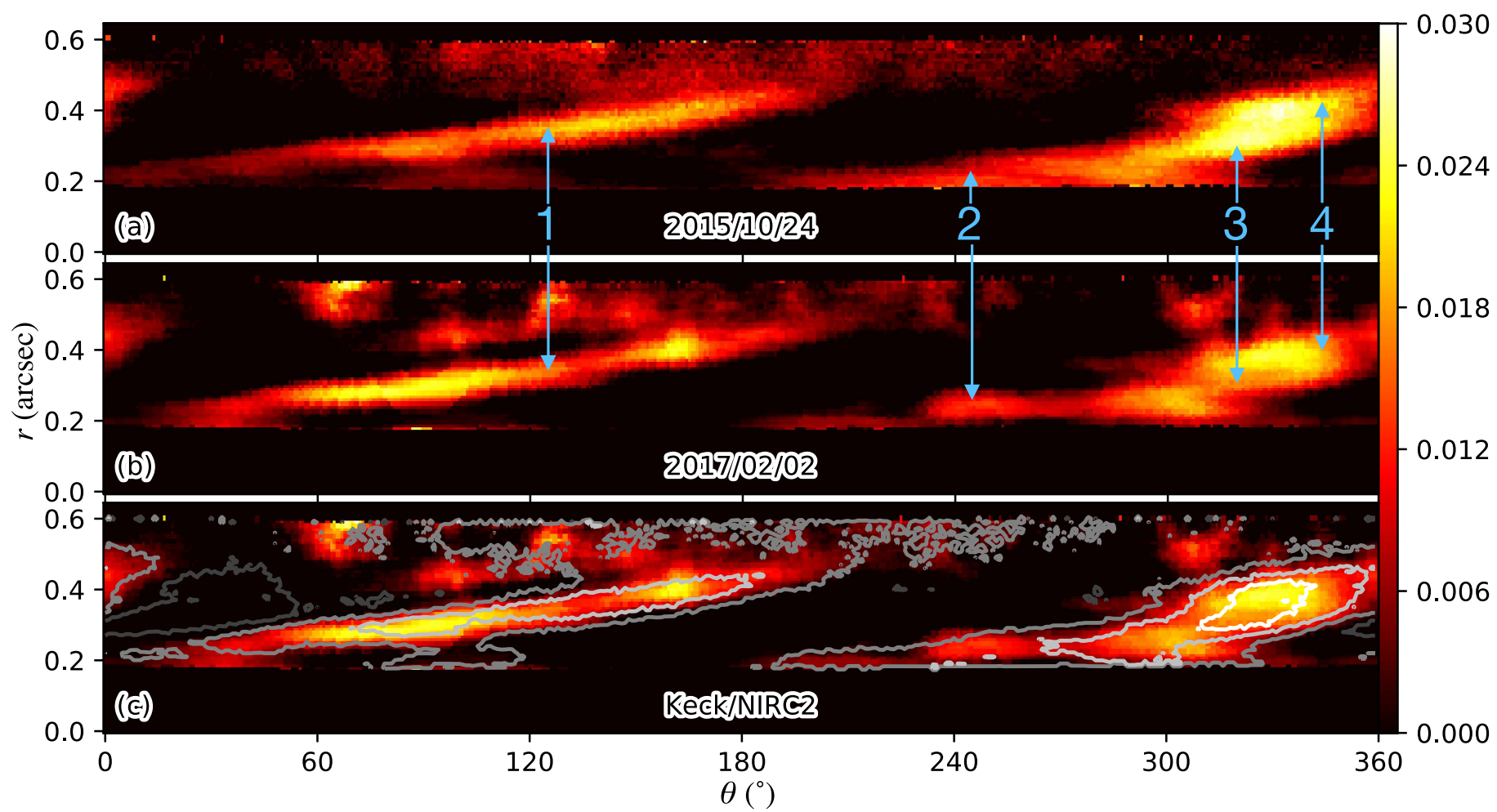

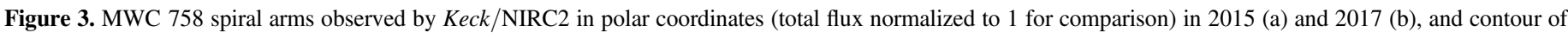
the 2015 observation overplotted on the 2017 one (c).

$\Delta \theta_{N}^{(1.28 \text { year })}=-0.70 \pm 6.78$, respectively. Since spiral arms in disks are trailing patterns, the MWC 758 arms are expected to rotate in a clockwise direction, i.e., $\Delta \theta \geqslant 0$, we therefore adopt the constraints from the southern primary arm, $\dot{\theta}=\Delta \theta_{S}^{(1.28 \text { year })} /$ 1.28 year $=0.6 \pm 8.3 \mathrm{yr}^{-1}$, as the rotation of the two.

For a consistency check, we measure the rotation with another method: in polar coordinates, we obtain the crosscorrelation maps (Tonry \& Davis 1979), and measure $\dot{\theta}=$ $0.7 \pm 56.2 \mathrm{yr}^{-1}$. We adopt the value from the LSDV method, since it is less biased by the nonspiral structures in the entire field of view as the cross-correlation method, and the best-fit values agree within 0 . 1 .

\subsubsection{NICMOS versus 2015 SPHERE (10.17 years apart)}

By analyzing the rotation between the NICMOS and SPHERE images, we narrow down the uncertainty determined from the NIRC2 observations here. We adopt the NIRC2 best-fit and uncertainty values, rotate the SPHERE result back to the NICMOS epoch, then the two images are expected to have no azimuthal shift. By fitting identical profiles and offsets for the rotated SPHERE and original NICMOS observations, and given the northern arm is blended with its secondary arm (marked by "4" in Figure 3) but with a smaller uncertainty than the southern arm, we adopt the results from the southern arm, obtaining a conservative measurement of $\Delta \theta^{(10.17 \text { year })}=6^{\circ} .1 \pm 29^{\circ} .4$. This corresponds to a statistical uncertainty for the angular speed: $(\dot{\theta \theta})_{\text {statistical }}=29^{\circ} .4 / 10.17$ year $=2.89 \mathrm{yr}^{-1}$.

\subsection{Additional Systematics}

We identify and study the impact of two possible systematics associated with the NICMOS results: the alignment uncertainty of stellar center determination (CD), and the misalignment uncertainty of the star behind the focal plane mask (FPM).

To quantify the stellar CD uncertainty, we cross-correlate the raw MWC 758 exposures with the 814 ALICE references, and determine the $3 \sigma$ uncertainty to be 0.5 pixel along both horizontal and vertical directions. We then draw 1000 possible centers within \pm 0.5 pixel from the center determined by our Radon Transform method, and cross-correlate the arm images in polar coordinates with the SPHERE result, and obtain a $3 \sigma$ quantile of $(\Delta \theta)_{\mathrm{CD}}=0^{\circ} .6$. We therefore adopt a $3 \sigma$ upper limit of $(\dot{\theta})_{\mathrm{CD}}=0.6 / 10.17$ year $=0.06 \mathrm{yr}^{-1}$.

Since the arms lie near the edge of the NICMOS FPM, if they have rotated in this $\sim 10$ year span, with the star not well-centered on the FPM during the time of the observation, this may still yield nonsignificant moving spirals. To account for this, we simulate 1000 SPHERE images with the following two parameters: (1) rotations within $\pm 60^{\circ}$ (denoted as $\alpha$ ): a range that the arms would rotate in $\sim 10$ years if they are driven by the protoplanet candidate reported by Reggiani et al. (2017), and (2) shifted centers within \pm 0 ". 04 ( 0.5 NICMOS pixel) along both horizontal and vertical directions. We mask the resampled SPHERE data with a circle of the NICMOS FPM size, then cross-correlate them with the original SPHERE image, and obtain their relative azimuthal shift $\left(\Delta \theta^{\prime}\right)$, which is then subtracted by introduced shift $(\alpha)$. We obtain a $3 \sigma$ upper limit $(\Delta \theta)_{\mathrm{FPM}}=\Delta \theta^{\prime}-\alpha=11^{\circ}$. Therefore, $(\delta \dot{\theta})_{\mathrm{FPM}}=$ $11^{\circ} / 10.17$ year $=1.08 \mathrm{yr}^{-1}$.

\section{Result}

From the previous analyses of statistical and systematical uncertainties, we obtain a total uncertainty in the rotation of the 


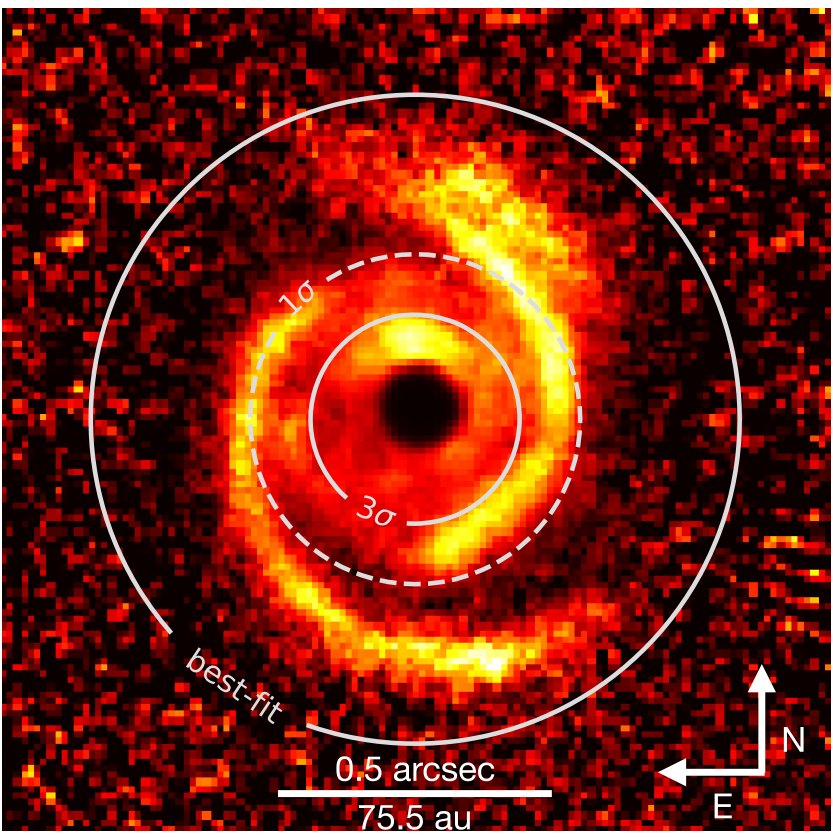

Figure 4. Best-fit and $3 \sigma$ lower limit (solid lines) of where the major-armdriving planets should be based on the measured pattern speed, plotted over the SPHERE data. The $1 \sigma$ limit (dashed line), which is interpolated from the bestfit and the $3 \sigma$ limit, is presented for the purpose of illustration and it may not represent a true $68 \%$ likelihood.

arms at $3 \sigma$ :

$$
\begin{aligned}
\delta \dot{\theta} & =\sqrt{(\delta \dot{\theta})_{\text {statistical }}^{2}+(\delta \dot{\theta})_{\text {systematic }}^{2}} \\
& =\sqrt{(\delta \dot{\theta})_{\text {statistical }}^{2}+\left[(\delta \dot{\theta})_{\mathrm{CD}}^{2}+(\delta \dot{\theta})_{\mathrm{FPM}}^{2}+(\delta \dot{\theta})_{\text {pixel }}^{2}\right]} \\
& =\sqrt{2.89^{2}+\left(0.06^{2}+1.08^{2}+1.18^{2}\right)}=3.31 \mathrm{yr}^{-1}
\end{aligned}
$$

where the pixel uncertainty is accounted for the NICMOS pixel size of $12^{\circ}$ at $r \sim 0$ " 6 (i.e., $(\delta \dot{\theta})_{\text {pixel }}=12^{\circ} / 10.17$ year $=$ $\left.1.18 \mathrm{yr}^{-1}\right)$. Together with the best-fit value, we obtain

$$
\dot{\theta}=0.6_{-0.6}^{+3.3} \mathrm{yr}^{-1}
$$

where the lower limit is physically constrained from the clockwise rotation of the MWC 758 arms.

For hypothesized arm-driving planet(s) on a circular orbit (eccentricity $e=0$ ), the best-fit pattern speed corresponds to a period of $T=598$ years, or a radial separation of $r_{\text {best }}=89 \mathrm{au}$; and the $3 \sigma$ upper limit leads to $T=92$ years and $r_{3 \sigma}=26 \mathrm{au}$; see Figure 4 for graphical representations.

For $e>0$, if the planet reaches its apogee in the current epoch, the limit on the arm motion translates into a stellocentric separation $r_{\min }(e)=(1-e)^{1 / 3} r$ au. For giant planets of several Jupiter mass forming in and interacting with a gaseous disk, their eccentricities are not expected to grow beyond $\sim 0.1$ (Dunhill et al. 2013; Duffell \& Chiang 2015). Furthermore, density waves excited by Jovian planets with $e \gtrsim 0.2$ start to deviate from their usual morphology, as the waves launched at different epochs interact with each other (H. Li \& S. Li 2018, private commnication), which provide poor fits to the arms around MWC 758. In this case, $e=0.2$ leads to a $7 \%$ decrease in the minimum stellocentric separation, and the hypothetical arm-driving planet(s) should be located at least 25 au from the star in 2017 at $3 \sigma$.
One might consider another scenario in which the two spiral arms exchanged positions between 2005 and 2015, i.e., rotated $\sim 180^{\circ}$. However, this means the major arms should have rotated an additional $22^{\circ} .6$ between the 2015 and 2017 NIRC2 observations, this is ruled out at $6 \sigma$ with our constraints.

\section{Summary and Discussion}

We present reprocessed 2005 HST/NICMOS observations of the MWC 758 disk, and successfully retrieve the two spiral arms in the system revealed by ground-based high-contrast imaging facilities. Thanks to the HST image, we are able to establish a 10 year baseline in observations to constrain the pattern speed of the major arms. Together with a 2015 VLT/ SPHERE data set, and two Keck/NIRC2 observations in 2015 and 2017, we obtain a rotation speed of $0.6_{-0.6}^{+3.3} \mathrm{yr}^{-1}$ at $3 \sigma$ for the two major spiral arms. The results correspond to a best-fit value of 89 au ( 0 ". 59), and a $3 \sigma$ lower limit of 26 au ( 0 !" 17), for the orbital distance of the hypothesized arm-driving perturber on a circular orbit.

Our constraint is consistent with the Dong et al. (2015b) prediction of the arm-driving planet at $\sim 90 \mathrm{au}\left(\sim 00^{\prime \prime} 6\right)$. In addition, we rule out at a $6 \sigma$ level the scenario that the companion candidate at 0 "! 11 (17 au) reported by Reggiani et al. (2017) is driving the major two spiral arms, assuming the candidate is on a circular orbit coplanar with the arms. This is further supported by Bae \& Zhu (2017), that a Jovian planet can drive only one external arm assuming a reasonable disk scale height. For new arm(s) and planet candidate(s) (e.g., Reggiani et al. 2017), more observations are needed to confirm their existence and dynamical connections.

The possible arm-driving planets in the MWC 758 system are excellent targets for future observations in direct imaging observations both from the ground and with the James Webb Space Telescope, and in millimeter observations using ALMA to search for evidence of a circumplanetary disk (e.g., Eisner 2015; Perez et al. 2015; Zhu 2015; Szulágyi et al. 2018).

We are grateful to the anonymous referee for constructive suggestions that improved our Letter, and Sean Brittain for insightful discussions. B.R. acknowledges the computational resources from the Maryland Advanced Research Computing Center (MARCC), which is funded by a State of Maryland grant to Johns Hopkins University through the Institute for Data Intensive Engineering and Science (IDIES). E.C. acknowledges support from NASA through Hubble Fellowship grant HST-HF251355 awarded by STScI, operated by AURA, Inc. under contract NAS5-26555, and support from HST-AR-12652, for research carried out at the Jet Propulsion Laboratory, California Institute of Technology. T.E. was supported in part by NASA Grants NNX15AD95G/NEXSS, NNX15AC89G, and NSF AST-1518332. This research has made use of data reprocessed as part of the ALICE program, which was supported by NASA through grants HST-AR-12652 (PI: R. Soummer), HST-GO11136 (PI: D. Golimowski), HST-GO-13855 (PI: E. Choquet), HST-GO-13331 (PI: L. Pueyo), and STScI Director's Discretionary Research funds, and was conducted at STScI, which is operated by AURA under NASA contract NAS5-26555. The input images to ALICE processing are from the recalibrated NICMOS data products produced by the Legacy Archive project, "A Legacy Archive PSF Library And Circumstellar Environments (LAPLACE) Investigation," (HST-AR-11279, PI: G. Schneider). This work benefited from NASA's Nexus for Exoplanet System 
Science (NExSS) research coordination network sponsored by NASA's Science Mission Directorate. The authors wish to recognize and acknowledge the very significant cultural role and reverence that the summit of Maunakea, host to the W. M. Keck Observatory, has always had within the indigenous Hawaiian community. We are most fortunate to have the opportunity to conduct observations from this mountain.

\section{Appendix}

\section{The LSDV Method}

When the morphological patterns of the spiral arms do not change among different epochs, the only difference is their relative azimuthal offset. This is known as the "fixed effect" in statistics, which has been extensively studied with the classical LSDV method. LSDV generalizes the ordinary least square (OLS) method using dummy variables. In this section, we first describe the classical LSDV method, then introduce our generalization of the method by taking into account the uncertainty from input data.

\section{A.1. The Classical LSDV Method}

In polar coordinates, the location of spiral arms can be represented by $\left(\theta_{\mathrm{PA}} \pm \delta \theta_{\mathrm{PA}}, r\right)$ pairs, which represents the position angle (with its uncertainty) and radial separation. For a given radial separation, its position angle can be estimated from fitting Gaussian profiles at different azimuthal directions. Assume there are $E$ epochs, each has $n$ data pairs, we can use a Taylor polynomial of degree $p$ to represent the arm morphology (e.g., Grady et al. 2013; Benisty et al. 2015; Reggiani et al. 2017). The classical LSDV method finds for all the data the best fit of

$$
\theta_{\mathrm{PA}, i}=f\left(r_{i}\right)=\sum_{j=1}^{p} c_{j} r_{i}^{j}+\sum_{k=1}^{E} d_{k} D_{k}(i),
$$

where the dummy variables $D_{k}(i)=1$ only when the $\left(\theta_{\mathrm{PA}, i}, r_{i}\right)$ pair is obtained from epoch $k$, and 0 otherwise. The coefficients $d$ are then the position angles of the spiral arms when $r=0$.

Let set $\mathbb{R}^{s \times t}$ contain $s$-by- $t$ real-valued matrices, if we denote the $\theta_{\mathrm{PA}}$ 's by $\boldsymbol{\Theta} \in \mathbb{R}^{n E \times 1}$, with $\boldsymbol{\Theta}_{i}=\theta_{\mathrm{PA}, i}$; the $r$ 's and dummy variables by $\boldsymbol{R} \in \mathbb{R}^{n E \times(p+E)}$, with $\boldsymbol{R}_{i(\cdot)}=$ $\left[r_{i}, r_{i}^{2}, \cdots, r_{i}^{p}, D_{1}(i), D_{2}(i), \cdots, D_{E}(i)\right] ;$ and the coefficients $\boldsymbol{\beta}=\left[c_{1}, \cdots, c_{p}, d_{1}, \cdots, d_{E}\right]^{T} \in \mathbb{R}^{(p+E) \times 1}$. We now write Equation (2) in a matrix OLS form:

$$
\Theta=\boldsymbol{R} \boldsymbol{\beta}+\boldsymbol{\epsilon},
$$

where $\epsilon \in \mathbb{R}^{n E \times 1}$ is the residual. Its cost function,

$$
\begin{aligned}
C(\boldsymbol{\Theta}, \boldsymbol{R} ; \boldsymbol{\beta}) & =\boldsymbol{\epsilon}^{T} \boldsymbol{\epsilon} \\
& =(\boldsymbol{\Theta}-\boldsymbol{R} \boldsymbol{\beta})^{T}(\boldsymbol{\Theta}-\boldsymbol{R} \boldsymbol{\beta}) \\
& =\sum_{i=1}^{n E}\left[\theta_{\mathrm{PA}, i}-\left(\sum_{j=1}^{p} c_{j} r_{i}^{j}+\sum_{k=1}^{n E} d_{k} D_{k}(i)\right)\right]^{2},
\end{aligned}
$$

is minimized by

$$
\hat{\boldsymbol{\beta}}=\left(\boldsymbol{R}^{T} \boldsymbol{R}\right)^{-1} \boldsymbol{R}^{T} \boldsymbol{\Theta},
$$

where ${ }^{T}$ and ${ }^{-1}$ stand for matrix transpose and inverse. The standard deviations of $\hat{\boldsymbol{\beta}}$ are calculated from the element-wise square root of the diagonal elements in the variance-covariance matrix:

$$
\begin{aligned}
\delta \hat{\boldsymbol{\beta}} & =\sqrt{\delta^{2} \hat{\boldsymbol{\beta}}}=\sqrt{\operatorname{diag}\left\{E\left[(\hat{\boldsymbol{\beta}}-\boldsymbol{\beta})(\hat{\boldsymbol{\beta}}-\boldsymbol{\beta})^{T}\right]\right\}} \\
& =\sqrt{\operatorname{diag}\left\{\hat{\sigma}^{2}\left(\boldsymbol{R}^{T} \boldsymbol{R}\right)^{-1}\right\}},
\end{aligned}
$$

where $\hat{\sigma}^{2}=\frac{(\boldsymbol{\Theta}-\boldsymbol{R} \hat{\boldsymbol{\beta}})^{T}(\boldsymbol{\Theta}-\boldsymbol{R} \hat{\boldsymbol{\beta}})}{n E-(p+E)}$.

\section{A.2. The Weighted LSDV Method}

To take into account the measurement uncertainty in our study, we generalize the classical LSDV method into a weighted form. The weighted LSDV method minimizes the chi-squared statistic:

$$
\begin{aligned}
\chi^{2}(\boldsymbol{\Theta}, \boldsymbol{R} ; \boldsymbol{\beta}) & =\left(\frac{\boldsymbol{\Theta}-\boldsymbol{R} \boldsymbol{\beta}}{\delta \boldsymbol{\Theta}}\right)^{T}\left(\frac{\boldsymbol{\Theta}-\boldsymbol{R} \boldsymbol{\beta}}{\delta \boldsymbol{\Theta}}\right) \\
& =\sum_{i=1}^{n E}\left[\frac{\theta_{\mathrm{PA}, i}}{\delta_{\theta_{\mathrm{PA}, i}}}-\left(\sum_{j=1}^{p} c_{j} \frac{r_{i}^{j}}{\delta_{\theta_{\mathrm{PA}, i}}}+\sum_{k=1}^{n E} d_{k} \frac{D_{k}(i)}{\delta_{\theta_{\mathrm{PA}, i}}}\right)\right]^{2},
\end{aligned}
$$

where the division operation is element-wise; and $\delta \Theta \in \mathbb{R}^{n E \times 1}$ stores the uncertainty for $\Theta$. With substitution

$$
\left\{\begin{array}{l}
\theta_{\mathrm{PA}, i}^{\prime}=\frac{\theta_{\mathrm{PA}, i}}{\delta_{\theta_{\mathrm{PA}, i}}} \\
r_{i}^{\prime j}=\frac{r_{i}^{j}}{\delta_{\theta_{\mathrm{PA}, i}}} \\
D^{\prime}{ }_{k}(i)=\frac{D_{k}(i)}{\delta_{\theta_{\mathrm{PA}, i}}},
\end{array}\right.
$$

where ' denotes the (element-wise) division of $\delta \theta_{\mathrm{PA}, i}$, we have a matrix form of

$$
\begin{aligned}
\chi^{2}(\boldsymbol{\Theta}, \boldsymbol{R} ; \boldsymbol{\beta}) & =\sum_{i=1}^{n E}\left[\theta_{\mathrm{PA}, i}^{\prime}-\left(\sum_{j=1}^{p} c_{j} r_{i}^{\prime j}+\sum_{k=1}^{n E} d_{k} D^{\prime}{ }_{k}(i)\right)\right]^{2} \\
& =\left(\boldsymbol{\Theta}^{\prime}-\boldsymbol{R}^{\prime} \boldsymbol{\beta}\right)^{T}\left(\boldsymbol{\Theta}^{\prime}-\boldsymbol{R}^{\prime} \boldsymbol{\beta}\right) \\
& =C\left(\boldsymbol{\Theta}^{\prime}, \boldsymbol{R}^{\prime} ; \boldsymbol{\beta}\right),
\end{aligned}
$$

as in Equation (4), whose best-fit values and standard deviations can thus be obtained from Equations (5) and (6).

With two epochs of observations in our fitting, we have $E=2$ and focus only on the difference of the last two terms in $\hat{\boldsymbol{\beta}}$, i.e., $\Delta d=\hat{d}_{1}-\hat{d}_{2}$, as well as the uncertainty $\delta(\Delta d)=$ $\sqrt{\delta^{2} \hat{d}_{1}+\delta^{2} \hat{d}_{2}-2 \operatorname{Cov}\left(\hat{d}_{1}, \hat{d}_{2}\right)}$. In our fitting efforts, we investigated Taylor polynomials up to $p=3$ degrees to study the different morphological pattern of the spiral arms; however, no significant difference was observed, we therefore only report the linear results in this Letter.

\section{ORCID iDs}

Bin Ren (任涁) (1D https://orcid.org/0000-0003-1698-9696 Ruobing Dong (董若冰) (10 https://orcid.org/0000-0001-9290-7846 Thomas M. Esposito (iD https://orcid.org/0000-00020792-3719

John H. Debes (iD https://orcid.org/0000-0002-1783-8817 Charles A. Poteet (iD https://orcid.org/0000-0003-4845-7483 Élodie Choquet (iD https://orcid.org/0000-0002-9173-0740 
Myriam Benisty (ib https://orcid.org/0000-0002-7695-7605

Eugene Chiang (i) https://orcid.org/0000-0002-6246-2310

Dean C. Hines 다 https://orcid.org/0000-0003-4653-6161

Glenn Schneider (10) https://orcid.org/0000-0002-4511-5966

Rémi Soummer (1) https://orcid.org/0000-0003-2753-2819

\section{References}

Akiyama, E., Hashimoto, J., Liu, H. B., et al. 2016, AJ, 152, 222 Andrews, S. M., Wilner, D. J., Espaillat, C., et al. 2011, ApJ, 732, 42 Bae, J., \& Zhu, Z. 2017, arXiv: 1711.08166

Bae, J., Zhu, Z., \& Hartmann, L. 2016, ApJ, 819, 134

Baraffe, I., Homeier, D., Allard, F., \& Chabrier, G. 2015, A\&A, 577, A42

Benisty, M., Juhasz, A., Boccaletti, A., et al. 2015, A\&A, 578, L6

Benisty, M., Stolker, T., Pohl, A., et al. 2017, A\&A, 597, A42

Choquet, É, Pueyo, L., Hagan, J. B., et al. 2014, Proc. SPIE, 9143, 914357

Clampin, M., Krist, J. E., Ardila, D. R., et al. 2003, AJ, 126, 385

Dong, R., \& Fung, J. 2017, ApJ, 835, 146

Dong, R., Fung, J., \& Chiang, E. 2016a, ApJ, 826, 75

Dong, R., Hall, C., Rice, K., \& Chiang, E. 2015a, ApJL, 812, L32

Dong, R., Zhu, Z., Fung, J., et al. 2016b, ApJL, 816, L12

Dong, R., Zhu, Z., Rafikov, R. R., \& Stone, J. M. 2015b, ApJL, 809, L5

Duffell, P. C., \& Chiang, E. 2015, ApJ, 812, 94

Dunhill, A. C., Alexander, R. D., \& Armitage, P. J. 2013, MNRAS, 428, 3072

Eisner, J. A. 2015, ApJL, 803, L4

Follette, K. B., Rameau, J., Dong, R., et al. 2017, AJ, 153, 264

Fung, J., \& Dong, R. 2015, ApJL, 815, L21

Gaia Collaboration, Prusti, T., de Bruijne, J. H. J., et al. 2016, A\&A, 595, A1

Garufi, A., Quanz, S. P., Avenhaus, H., et al. 2013, A\&A, 560, A105

Grady, C. A., Muto, T., Hashimoto, J., et al. 2013, ApJ, 762, 48

Hagan, J. B., Choquet, E., Soummer, R., \& Vigan, A. 2018, arXiv:1802.07754
Huby, E., Bottom, M., Femenia, B., et al. 2017, A\&A, 600, A46 Isella, A., Natta, A., Wilner, D., Carpenter, J. M., \& Testi, L. 2010, ApJ, 725,1735

Kley, W., \& Nelson, R. P. 2012, ARA\&A, 50, 211

Konishi, M., Grady, C. A., Schneider, G., et al. 2016, ApJL, 818, L23

Kratter, K., \& Lodato, G. 2016, ARA\&A, 54, 271

Lodato, G., \& Rice, W. K. M. 2005, MNRAS, 358, 1489

Lomax, J. R., Wisniewski, J. P., Grady, C. A., et al. 2016, ApJ, 828, 2

Maire, A.-L., Stolker, T., Messina, S., et al. 2017, A\&A, 601, A134

Marois, C., Lafrenière, D., Doyon, R., Macintosh, B., \& Nadeau, D. 2006, ApJ, 641, 556

Meeus, G., Montesinos, B., Mendigutía, I., et al. 2012, A\&A, 544, A78

Mouillet, D., Lagrange, A. M., Augereau, J. C., \& Ménard, F. 2001, A\&A, 372, L61

Muto, T., Grady, C. A., Hashimoto, J., et al. 2012, ApJL, 748, L22

Perez, S., Casassus, S., Ménard, F., et al. 2015, ApJ, 798, 85

Poteet, C. A., Chen, C. H., Hines, D. C., et al. 2018, ApJ, submitted

Pueyo, L., Soummer, R., Hoffmann, J., et al. 2015, ApJ, 803, 31

Reggiani, M., Christiaens, V., Absil, O., et al. 2017, arXiv:1710.11393

Ren, B., Pueyo, L., Zhu, G. B., Debes, J., \& Duchêne, G. 2018, ApJ, 852, 104

Serabyn, E., Huby, E., Matthews, K., et al. 2017, AJ, 153, 43

Service, M., Lu, J. R., Campbell, R., et al. 2016, PASP, 128, 095004

Siess, L., Dufour, E., \& Forestini, M. 2000, A\&A, 358, 593

Soummer, R., Pueyo, L., \& Larkin, J. 2012, ApJL, 755, L28

Stolker, T., Dominik, C., Avenhaus, H., et al. 2016, A\&A, 595, A113

Szulágyi, J., van der Plas, G., Meyer, M. R., et al. 2018, MNRAS, 473, 3573

Tonry, J., \& Davis, M. 1979, AJ, 84, 1511

van der Marel, N., Verhaar, B. W., van Terwisga, S., et al. 2016, A\&A, 592, A126

Wagner, K., Apai, D., Kasper, M., \& Robberto, M. 2015, ApJL, 813, L2

Wagner, K., Dong, R., Sheehan, P., et al. 2018, ApJ, 854, 130

Zhu, Z. 2015, ApJ, 799, 16

Zhu, Z., Dong, R., Stone, J. M., \& Rafikov, R. R. 2015, ApJ, 813, 88 